UCRL-ID-119734

\title{
LLNL State-of-the-Art Codes for Source Calculations
}

\author{
Lewis A. Glenn
}

This paper is submitted as

CTBT Seismic Research FY95 Deliverable No. 7(S6.1)

February 1995

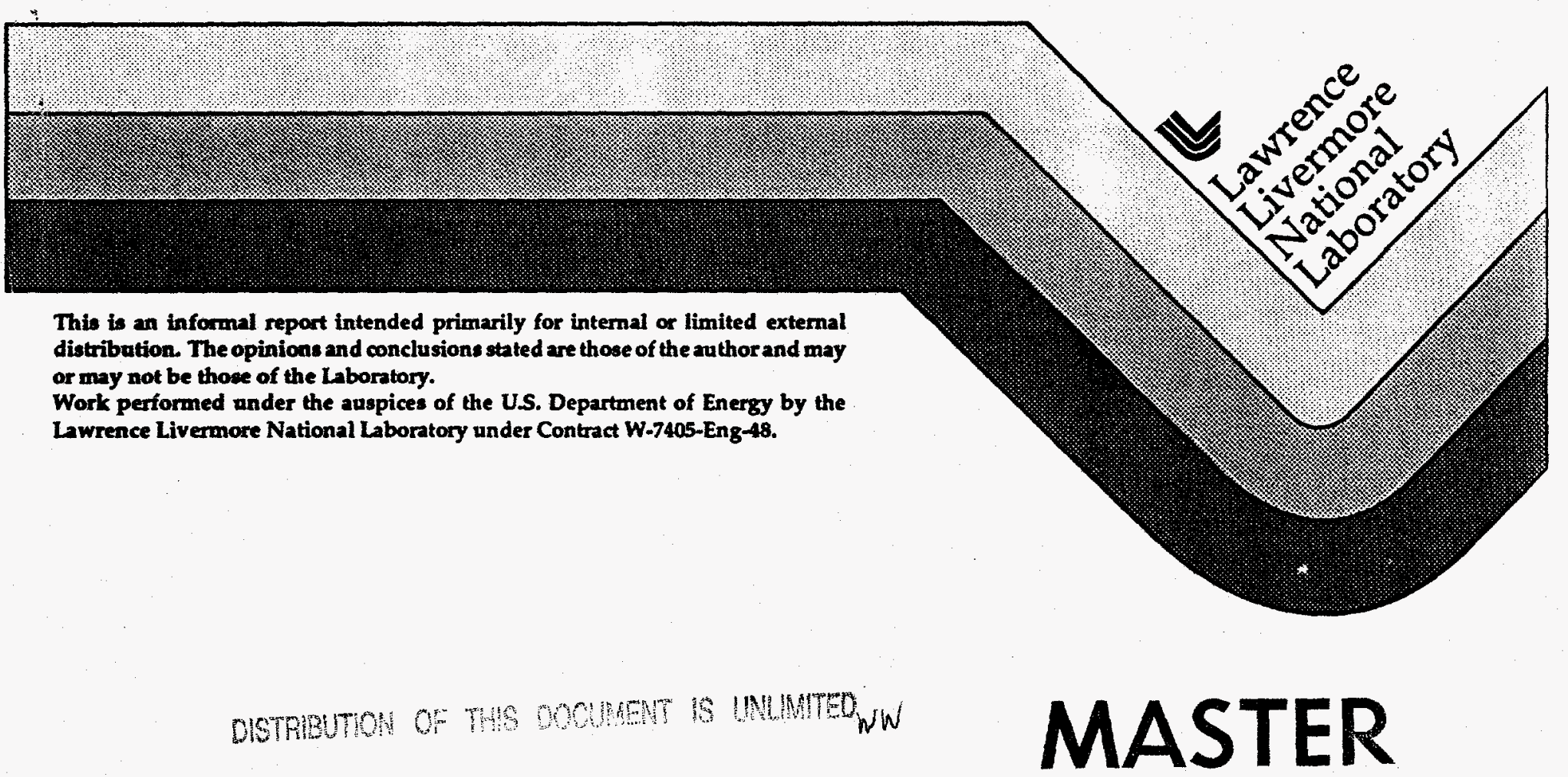




\section{DISCLAIMER}

This report was prepared as an account of work sponsored by an agency of the United States Government. Neither the United States Government nor any agency thereof, nor any of their employees, make any warranty, express or implied, or assumes any legal liability or responsibility for the accuracy, completeness, or usefulness of any information, apparatus, product, or process disclosed, or represents that its use would not infringe privately owned rights. Reference herein to any specific commercial product, process, or service by trade name, trademark, manufacturer, or otherwise does not necessarily constitute or imply its endorsement, recommendation, or favoring by the United States Government or any agency thereof. The views and opinions of authors expressed herein do not necessarily state or reflect those of the United States Government or any agency thereof. 


\section{DISCLAIMER}

Portions of this document may be illegible in electronic image products. Images are produced from the best available original document. 


\section{Introduction}

The explosion-source region is defined as the region surrounding an underground explosion that cannot be described by elastic or anelastic theory. This region extends typically to ranges on the order of $1 \mathrm{~km} / \mathrm{kt}^{1 / 3}$. For the simulation or analysis of seismic signals, what is required is the time resolved motion and stress state at the inelastic boundary. Various analytic approximations have been made for these boundary conditions, but since they rely on near-field empirical data they cannot be expected to reliably extrapolate to different explosion sites. More important, without some knowledge of the initial energy density and the characteristics of the medium immediately surrounding the explosion, these simplified models are unable to distinguish chemical from nuclear explosions, identify cavity decoupling, or account for such phenomena as anomalous dissipation via pore collapse.

Our purpose here is to document the state-of-the-art codes at LLNL involved in simulating underground (chemical and nuclear) explosions and, in so doing, present an overview of the physics. In what follows, we first describe the fundamental equations involved, discuss solution methods, coordinate frames and dimensionality. Then we identify the codes used at LLNL and their limitations. A companion report will describe the factors that most influence the seismic response, i.e., the source properties important for discrimination. That report will emphasize the coupling between the rock properties and the characteristics of the explosion cavity.

\section{Fundamental Equations}

In divergence form, the equations describing the conservation of mass, momentum, and total energy respectively are:

$$
\begin{aligned}
& \frac{1}{\rho} \frac{D \rho}{d t}=-\nabla \cdot \mathrm{u} \\
& \rho \frac{\mathrm{Du}}{d t}=-\nabla\left(p+p_{R}\right)+\nabla \cdot \sigma^{\prime}+\mathrm{f} \\
& \rho \frac{D e}{d t}=-\nabla \cdot\left[\mathrm{u}\left(p+p_{R}\right)\right]+\nabla \cdot\left(\mathrm{u} \bullet \sigma^{\prime}\right)+\nabla \cdot\left[\left(\kappa+\kappa_{R}\right) \nabla T\right]+Q
\end{aligned}
$$

where $\rho$ is the mass density, $u$ the particle velocity vector, $p$ the material pressure, 


\section{LLNL State-of-the-Art Codes for Source Calculations}

$\sigma^{\prime}=\sigma-p I$ is the deviatoric stress tensor (I being the unit tensor), and $f$ the body-force per unit volume (gravity) vector. $p_{R}=a_{R} T^{4 / 3}$ is the radiation pressure, where $a_{R}$ is the radiation density constant $\left(7.563 \times 10^{-15} \mathrm{erg} / \mathrm{cm}^{3} /\left({ }^{\circ} \mathrm{K}\right)\right)$ and $T$ is the temperature.

$e=e_{m}(\rho, T)+(1 / 2) u \bullet u+a_{R} T^{4} / \rho$ is the total specific internal energy. The first term in the latter expression, $e_{m}$, represents the material specific internal energy, the second term the specific kinetic energy, and the last term the specific energy associated with the radiation field. $K$ is the linear coefficient of thermal conductivity and $K_{R}=4 D_{R} a_{R} T^{3}$, where $D_{R}$ is the Rosseland radiation diffusion coefficient. $Q$ is the rate at which energy is generated by the explosive device per unit volume.

It is important to note that equations (1) - (3) are not closed until an equation of state, $p=p(\rho, T, \ldots)$ and a constitutive model $\sigma=\sigma(\rho, T, \varepsilon, \ldots)$ are defined. Here, $\varepsilon$ is the strain tensor and the continuation inside the parenthesis is meant to imply that path dependence, porosity, and rate effects may also be involved. The closure equations serve to define the medium. At high temperature, $\sigma^{\prime}$ vanishes and the equation of state is all that is required, and this may be very complex as in the case of rock material in the immediate vicinity of an explosive device; it is often the case that tables of the form $p=p(\rho, T, \ldots)$ are used. For rock media below the melting point, both the equation of state and the constitutive model may be complex. For example, consider a porous, dry rock. A very simple model for this kind of material was developed by Carroll and Holt [1972] and is widely used:

$p(v, e)=p_{m}(v / \alpha, e) / \alpha=p_{m}\left(v_{m}, e_{m}\right) / \alpha$

and

$\alpha=\alpha(p)$

where $v=1 / \rho$ is the specific volume, and the subscript ${ }_{m}$ refers to the matrix material in which the pores are embedded. The parameter $\alpha$ is related to the gas porosity, $\Psi$, and is defined by the relation

$$
\alpha \equiv v / v_{m}=\rho_{m} / \rho=(1-\Psi)^{-1}
$$

At ambient pressure, $\alpha=\alpha^{0}$, the initial value, and at some high pressure, $p_{c}$, the pores will be completely crushed and $\alpha\left(p_{c}\right)=1$. For $p>p_{c}$, a Grüneisen form for $p_{m}$ is normally employed in equation (4) and smoothly transitions into the tabular form $p_{m}(\rho, T)$.

Equations (5) and (6) apply to loading for $p<p_{\circ}$ but to unloading only when $p<p_{e l}$; in this small, but important range the pore volume behaves in strictly elastic fashion. In the range $p_{a l} \leq p<p_{c}$, unloading occurs at constant gas porosity. 
In the simple, porous rock model, the compressive strength depends on the pressure, temperature, and on a damage parameter that measures the degree of degradation due to tensile fracture. Specifically,

$$
\sqrt{3 J_{2}} \leq Y=(1-D) \bar{Y}+\beta D \bar{Y}
$$

where $j_{2}^{\prime}=(1 / 2) \sigma^{\prime} \bullet \sigma^{\prime}, \beta$ is a constant, typically 0.25 , and $D$ is a scalar function of the volumetric component of the void strain tensor (Rubin and Attia, 1990); typically, $D=\varepsilon_{i i}^{v} / \varepsilon_{\max }^{v}$ and $\varepsilon_{\max }^{v}$ is of the order of .005. The generalized compressive strength is:

$\bar{Y}=\left[Y_{0}+\frac{p}{a+\left(\frac{p}{Y_{u}-Y_{0}}\right)}\right] \max \left[\left(1-T / T_{m}\right)^{b}, 0\right]$

$Y_{O}$ is the unconfined compressive strength, $Y_{u}$ is the ultimate compressive strength, and $T_{m}(p)$ is the melting temperature. The parameters $a$ (the cohesion) and $b$ are constants. For porous rock media which are partly or completely saturated, more complex effective stress models are available.

We stress that equations (4) - (8) merely exemplify the models that are available for describing the response of the rock media. Many other models are available, and include detailed descriptions of multidimensional fracture, effective stress due to partial or complete saturation, multiple phase change, and rate effects. Also, equations of state have been developed for virtually all chemical explosives, inert gases, and nuclear-explosive components.

\section{Explosion Phases}

Simulating the explosion can be divided into 3 phases, since not all the terms in the above equations are significant at the same time. This allows operator splitting to be employed, which can simplify the calculations; for multidimensional problems, different codes are often used for each phase. The first phase we call the radiation-coupled hydrodynamic phase, the duration of which is typically: $0<t<10 \mu \mathrm{s}$. In this period the pressure is so large that strength and overburden effects can be ignored, deleting the last 2 terms on the right-hand-side (RHS) of equation (2) and the 2 nd term on the RHS of equation (3). We note that radiation transport is normally significant only when aspherical sources are employed or when large (decoupling) cavities are to be simulated; in the latter case the radiation diffuses ahead of the shock front and thereby mitigates the hydrodynamic impulse delivered to the wall. The diffusion approximation for the radiation transport is valid so long as the medium is optically thick. When photon mean free paths become 
comparable to characteristic structural lengths, such as the cavity radius, a flux limiter is employed to restrict the energy transport to physically allowable values.

The hydrodynamics phase occurs in the interval $10 \mu s \leq t \leq 1 \mathrm{~ms} / \mathrm{kt}^{1 / 3}$. In this interval, not only are strength and overburden negligible, but all radiation effects are as well. The pressure is determined strictly by the material equation of state.

The final phase, in which $t>1 \mathrm{~ms} / \mathrm{kt}^{1 / 3}$, we identify as strength-coupled hydrodynamics. By this point, the peak pressure has decreased typically below $10 \mathrm{GPa}$. The equation of state and the overall constitutive model may be quite complex. The pressure may exhibit strain-path and/or porosity dependence and the in situ yield strength is determined by the (overburden) pressure, porosity, and the degree of water saturation. The subsequent yield strength (on loading and unloading) may be enhanced by pressure and strain hardening, or degraded by damage and thermal softening. Morover, the initial overburden state may deviate significantly from lithostatic, an important consideration when explosions take place in large cavities.

\section{Coordinate Frames}

The usual procedure for solving source-region problems is to difference equations (1) - (3) directly in the time domain. Explicit time differencing, where state variables are updated strictly from their values at earlier time, is normally employed because of the ease of implementation and computational speed. An exception is made when considering the radiation diffusion term in equation (3), where (unconditionally stable) fully implicit schemes are the rule. The reason is that the Courant time step required for stable solution of the explicit radiation operator is many orders of magnitude less than for the coupled hydrodynamic system.

The mesh or grid employed for spatial differencing can be either structured, with fixed connectivity, or unstructured. Three kinds of structured grids have been used extensively. To briefly review them, consider the convective operator:

$$
\frac{D}{\partial t} \equiv \frac{\partial}{\partial t}+w \cdot \nabla
$$

where $\mathbf{w}=\mathbf{u}-\mathbf{s} ; \mathbf{u}$ is the particle velocity vector and $\mathbf{s}$ is the local coordinate frame velocity vector. If $\mathrm{u}$ is everywhere $=s, w=0$ and we have a Lagrangian coordinate frame, in which the advection operator is eliminated altogether. Although this simplifies the solution, Lagrangian frames normally cannot be used throughout because of mesh tangling problems and because the time step required for stable solution may become infinitesimal. When this occurs, a rezoning or dezoning operation is required, which amounts to overlaying a new grid on the old and interpolating the field variables thereon. 
Alternately, if $s$ is everywhere $=0, w=u$, and we have an Eulerian frame in which the mesh is stationary. In practice, this is usually accomplished in 2 steps. First the Lagrangian operator is employed to compute the motion in the material frame; the grid points are then moved back to their original position and the advection operator is used to remap the field quantities. Eulerian frames permit accurate spatial differences to be computed and are the easiest to visualize. Their disadvantage is that they require interpolation to define moving interfaces and tend to diffuse information across these interfaces. Moreover, they are inefficient in that the resolution of moving fronts, such as shock waves, requires the mesh density to be fine everywhere since the direction of wave motion is not normally known in advance. This problem can sometimes be alleviated by employing a multigrid scheme, in which all wave fronts are tracked, and fine grids are superimposed only at the fronts.

In the most general case, $s$ and $w$ can be adapted to the problem at hand; in this case the scheme is called ALE (arbitrary Lagrangian-Eulerian). ALE schemes allow interfaces to remain Lagrangian while the mesh is continuously deformed elsewhere. Various algorithms have been employed for optimizing the mesh motion.

Methods that use unstructured grids can theoretically eliminate mesh tangling problems without introducing excessive diffusion. They fall into two categories, distinguished primarily by the means employed for calculating the spatial derivatives. In the Free Lagrange formulation, nearest neighbor clustering of mass points is used to obtain standard finite difference approximations for the gradient and divergence of state variables. In the Smoothed Particle Hydrodynamics scheme, Monte Carlo theory is applied to the discrete representation of mass points in order to obtain a continuous representation of the state variables; analytical differentiation then gives the required spatial derivatives. Unstructured-grid methods have not been used at LLNL for seismicsource simulation because these techniques require fairly complex rules for associating points and, at least to date, are fairly slow compared with conventional structured formulations.

\section{Dimensionality}

Most source-region problems that generate boundary conditions for seismic propagation can be solved in 1 dimension, and assuming spherical symmetry. Typically, from $10^{2}$ to $10^{4}$ nodes are employed, depending on the required spatial resolution and the simulations can be carried out on a workstation in a few minutes to a few hours. When complicated topography or stratification is important, say for accurate spectral representation, or to simulate interfacial effects such as spall, $2 D$ or even $3 D$ codes are required. $2 D$ problems are normally calculated with axial symmetry although plane strain and plane stress solutions are also possible. Typical simulations employ from $10^{4}$ to $10^{6}$ nodes and require from a few hours on a workstation to a few days on a supercomputer. Fully $3 D$ simulations that employ of the order of the order of $10^{7}$ nodes or less can be carried out 
on a supercomputer, and a few problems of this size have been run on massively parallel machines, however high-resolution $3 D$ problems are still mostly beyond the state-of-theart.

\section{Codes Employed at LLNL for Source Calculations}

The main $1 D$ code employed is AFTONRS (Glenn 1978, 1993). This is an ALE code with very special characteristics; the difference equations are so-called form-preserving analogs of the differential equations of motion (Trulio and Trigger, 1961). If the kinetic energy equation, obtained by taking the scalar product of the momentum equation (2) with the particle velocity vector $u$, is subtracted from the equation for total energy (3), the result is an exact expression of the First Law of thermodynamics. Following the identical procedure with the equivalent difference equations leads to a difference equation for the First Law which is in one-to-one correspondence with the differential result, with no terms of any order left over.

AFTONRS is also very flexible and modular. Virtually any type of equation of state and constitutive model can be employed in either analytical or tabular form, so that most of our parametric source studies have used this code. Radiation transport is implemeneted in the diffusion approximation and tabular opacity data may be used. For spherical explosion calculations, a special mesh-motion algorithm is available that maintains fine, smooth zoning around the periphery of the expanding cavity without compromising the time step. This is accomplished by continuously moving the mesh elements such that the ratio of the first rock zone thickness to the cavity radius is maintained constant (typically at a value of 0.01 or less) and the adjacent rock zones grow in geomertic progression (typically with ratio factor $<1.005$ ). Moreover, all this is accomplished in less than 3500 lines of FORTRAN source code. Currently, AFTONRS runs on any UNIX machine, and can easily be adapted for PC useage.

For $2 D$ calculations, there are two choices. KDYNA (Levatin et al., 1990) is a $2 D$ Lagrangian code that is based on DYNA2D, arguably the most widely used $2 D$ continuum mechanics program in the world. DYNA2D was developed by Jon Hallquist at LLNL in the late 1970s (Hallquist, 1978). The KDYNA modifications include the provision for specification of lithostatic overburden in complex geologies, a dezoning procedure for calculating explosions, many new equations of state and constitutive models (of earth materials), and new input/output routines. KDYNA has been extensively employed for calculation of the containment potential of underground nuclear explosions in varying geologies. Its major limitation is the requirement for periodic dezoning of the rock region surrounding the expanding explosion cavity; special scripts are needed to perform this function and the necessarily disjoint fashion in which this is accomplished is a source of indeterminate error. Also, KDYNA does not handle strong shocks in the cavity gas region in an efficient manner, so that, for example, calculations of decoupling in aspherical cavities cannot be performed with this code. 
CALE (Tipton, 1987) is the code of choice for $2 D$ calculations of explosions and is rapidly supplanting KDYNA for this purpose. As the name suggests, CALE is an ALE code, and is written entirely in the $C$ programming language for maximum portability. It has been widely used for ordnance applications and is well suited for calculation of explosion sources. Its primary limitation, in comparison with KDYNA, is the relative paucity of rock-material models and lack of a sophisticated treatment of overburden. Both of these problems are currently being addressed and we anticipate that CALE will have most of the capabilities of KDYNA by the end of FY95.

$3 D$ calculations are generally not required for source calculations, except where questions of large azimuthal variations are involved, and usually these questions can be resolved with elastic codes (not addressed in this report). Exceptions include accounting for the nonlinear interaction of arrays of cylindrical charges near a quarry bench or corner, certain mine collapses, and perhaps decoupling in a room-and-pillar mine configuration. For these applications, two codes are available. The first, CTH, developed at Sandia National Laboratory (McGlaun et al., 1990), is an Eulerian code that had found wide useage both within and without the DOE network. CTH is highly portable, and runs on virtually all UNIX machines and supercomputers; there is even a version explicitly designed for massively parallel multiprocessors. The major disadvantage of CTH is that, like CALE (and even more so), relatively few rock-material models have been implemented. Also, although very easy to use, the code suffers from the usual problems of numerical diffusion associated with stricly Eulerian coordinate frames. The alternative to CTH is ALE3D (Couch et al., 1993), a 3D ALE code that is based on the Lagrangian framework of DYNA3D (Hallquist, 1981). Like CTH, ALE3D runs under the UNIX operating system, however this code has not been widely distributed and is still considered under development. Nevertheless, a number of source calculations have already been run with ALE3D to model explosions in tunnels and in quarries with multiple cylindrical charges. We have implemented the main constitutive models of rock media that were in KYDNA (extended to $3 D$ ) and expect to further extend these models in the future.

All $3 D$ codes utilize extensive computer resources and, even with large modern high-speed memories, are limited in the resolution they can provide. We are developing software for mapping the near field (that may require modeling with $3 D$ inelastic codes, such as CTH or ALE3D) into boundary element, reflectivity, or other integral-representation codes for propagation of seismic signals.

\section{References}

Carroll, M. M. and A. C. Holt (1972). Suggested modification of the $p-\alpha$ model for porous materials, A. Appl. Phys., 43, No. 2, 759-761. 


\section{LLNL State-of-the-Art Codes for Source Calculations}

Couch, R., R. Sharp, and I. Otero (1993). Application of ALE techniques to metal forming simulations, American Society of Mechanical Engineers AMD-Vol. 180, Advanced computational methods for material modeling, Book No. $\mathrm{H} 00883$ (eds. D. J. Benson and R. J. Asaro).

Glenn, L. A. (1978). AFTON Revisited: an improved algorithm for numerical solution of initial value problems in continuum mechanics, University of California, Lawrence Lawrence Livermore National Laboratory Report UCRL-52512, July 20.

Glenn, L. A. (1993). Energy-density effects on seismic decoupling, J. Geophys. Res., 98, no. B2, 1933-1942.

Hallquist, J. O. (1978). DYNA2D - an explicit finite element and finite difference code for axisymmetric and plane strain calculations (user's guide), University of California, Lawrence Livermore National Laboratory Report UCRL-52429.

Hallquist, J. O. (1981). User's manual for DYNA3D and DYNAP (nonlinear dynamic analysis of solids in three dimensions), University of California, Lawrence Livermore National Laboratory Report UCRL-19156.

Levatin, J. L., A. V. Attia, and J. O. Hallquist (1990). KDYNA user's manual, University of California, Lawrence Livermore National Laboratory Report UCRL-ID-106104, September 28.

McGlaun, J. M., S. L. Thompson, L. N. Kmetyk, and M. G. Elrick (1990). A brief description of the three-dimensional shock wave physics code CTH, Sandia National Laboratory Report SAND89-0607.

Rubin, M. B. and A. V. Attia (1990). A continuum tensile failure model with friction, University of California, Lawrence Livermore National Laboratory Report UCRLID-104759, August 21.

Tipton, R. E. (1987). A $2 D$ Lagrange MHD Code, Proceedings of the fourth international conference on megagauss magnetic field generation and related topics, Plenum Press, New York (eds. C. M. Fowler, R. S. Caird, and D. J. Erickson).

Trulio, J. G. and K. R. Trigger (1961). Numerical solution of the one-dimensional hydrodynamic equations in an arbitrary time-dependent coordinate system, University of California, Lawrence Livermore National Laboratory Report UCRL-6522, July 19. 